\title{
Research on Fracture Characteristics of Machine-made Sand Concrete by White Light Digital Image Analysis in Frequency Domain
}

\author{
Xia Jitao ${ }^{1, \text { a }}$ Yang Xinwei ${ }^{2, b}$ Wang Zhentian ${ }^{1, c}$ Qu Dengdian ${ }^{1, d}$ Zhan Qifang $^{2, e}$ \\ ${ }^{1}$ Guangda Railway Management of China Railway $21^{\text {st }}$ Bureau Corporation, China \\ ${ }^{2}$ Shijiazhuang Institute of Railway Technology, China \\ ajitaoxia21@163.com byxw1111111@126.com cwangzhentian888@163.com dengdianqu@126.co \\ m ${ }^{\mathrm{e}} z q f 221 @ 126 . c o m$
}

Keywords: fracture characteristic, machine-made sand, white light digital image analysis in frequency domain

\begin{abstract}
The machine-made sand concrete has been widely used in building engineering and its fracture characteristic is a research focus in the world. White light digital image analysis in frequency domain has the advantages of whole-field, non-contact measurement and the simple measuring system and can obtain displacements by frequency domination correlation arithmetic. In this paper, the method was introduced to study fracture characteristics of machine-made sand concrete. The results show the usability of the method for studying fracture characteristics of machine-made sand concrete.
\end{abstract}

\section{Introduction}

Because of low cost and enough strength, the machine-made sand concrete has been widely used in building engineering and attracted many researchers to study its material behaviors which mainly includes mechanical and chemical behaviors[1-2]. But few papers show its fracture charactersitics which is very important for the safety of whole structure of building.

White light digit image analysis in frequency domain is based on white light speckle method and digital image coherent analysis in frequency domain. The images before and after deformation are recorded by CCD camera and analyzed in computer to obtain the displacements of measured fields[3-4].

Because of the advantages of this method, in this paper, white light digit image analysis in frequency domain was introduced to study fracture characteristics of machine-made sand concrete.

\section{Principle of white light digital image analysis in frequency domain}

CCD camera was used to record gray images before and after deformation and analyze them in computer to obtain the displacement information. A small region in images is considered that the displacements of the small region are equal. Let $\delta$ denote the gray distributions of one point. $\delta_{0}(x, y)$ is the location before deformation of specimen, $\delta_{1}(x, y)$ is the corresponding location after deformation and $\delta_{1}(x, y)=\delta_{0}(x+u, y+v)$ as shown in Fig.1. $d$ is the displacement vector, $\theta$ is included angle between $d$ and $x$ axle.

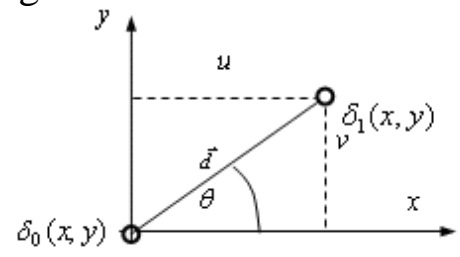

Fig.1 small region before and after deformation

$g$ is the sum of light intensity before and after deformation and we can obtain

$$
g(x, y)=\delta_{0}(x, y)+\delta_{0}(x+d \cos \theta, y+d \sin \theta)
$$


According to Eq. 1, Fourier transformation is used to obtain the spectrum

$$
G(X, Y)=2 G_{0}(X, Y)(1+\exp (-i \pi(2 \vec{r} . \vec{d}))
$$

where $\quad G_{0}(X, Y)=\mathfrak{I}\left\{\delta_{0}(x, y)\right\}=\iint \delta_{0}(x, y) \exp (-2 \pi i(x X+y Y)) d x d y=1 \quad, \quad \mathfrak{I}\{\} \quad$ is $\quad$ Fourier transformation, $(x, y)$ is the coordinate on object plane, $(X, Y)$ is the coordinate on spectrum plane. $\delta_{0}(x, y)$ and $G_{0}(X, Y)$ denote the gray distribution of small region in images and its Fourier transformation respectively. $\vec{r}$ is the coordinate vector on spectrum plane.

The distribution of light intensity on spectrum plane can be obtained

$$
I=G^{*} G=4 \cos ^{2}[\pi(\vec{r} \cdot \vec{d})]
$$

According to Eq.3, when $\pi(\vec{r} \cdot \vec{d})=n \pi, I$ can be its maximum; when $\pi(\vec{r} \cdot \vec{d})=(n-1 / 2) \pi, I$ is the minimum.

A series of parallel fringes can be shown in the form of image.

$$
\vec{r} \cdot \vec{d}=\left\{\begin{array}{ll}
n & \text { bright fringe } \\
n-\frac{1}{2} & \text { dark fringe }
\end{array} \quad(n=0, \pm 1, \pm 2, \ldots)\right.
$$

We consider that the displacement of every point in small region is equal. $N$ and $N^{\prime}=N+1$ denote fringe values of adjacent fringes and we have

$$
r_{N} \cdot d=N, r_{N+1} \cdot d=N+1 \text {. }
$$

So $d=(N+1)-N) /\left(r_{N+1}-r_{N}\right)$ and

$$
d=\frac{1}{\Delta r}
$$

where $\Delta r$ is the fringe separation.

Clearly, the displacement vector is vertical to the fringe direction and its magnitude can be obtained according to Eq. 5.

The imaging lens in CCD is used when images are recorded, so the real displacement $d_{T}$ is

where $M$ is image multiple.

$$
d_{T}=\frac{d}{M}=\frac{1}{M \Delta r}
$$

According to domination transformation theory, we have

$$
\Delta r=\frac{1}{L c c d} \Delta K
$$

where $L c c d$ is target dimension of CCD, $\Delta K$ is pixel number between the fringes.

Substitute Eq. 7 into Eq. 6 and we obtain

$$
d_{T}=\frac{L c c d}{M \cdot \Delta K}
$$

$u, v$ are the components of $d_{T}$ on $x, y$ axle respectively.

\section{Experiment}

The specimen is a rectangular machine-made sand concrete and its dimension is $160 \mathrm{~mm} \times 40 \mathrm{~mm} \times 40 \mathrm{~mm}$

The specimen is precracked and the length of crack is $6.2 \mathrm{~mm}$. The surface of the specimen was sprayed with speckle for enhancing its surface characteristics. It is fixed on the experimental machine and the loading method is three-point bending load as shown in Fig.2.

CCD camera used is MV-AD040SC and was placed before the specimen as shown in Fig.3. Before and after loading, the crack-tip field images were recorded by CCD camera. 


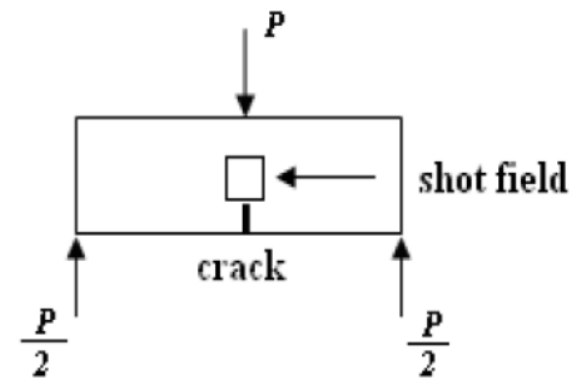

Fig.2 loading method

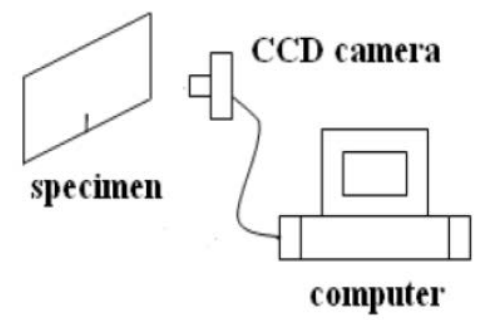

Fig.3 recording system

\section{Experimental results}

The images of crack-tip field before and after loading were recorded by CCD carema and input into computer as shown in Fig. 4

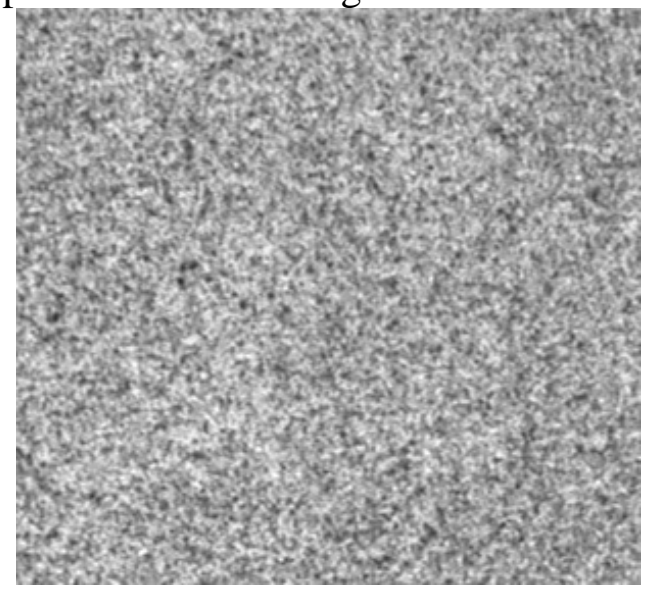

(a) before loading

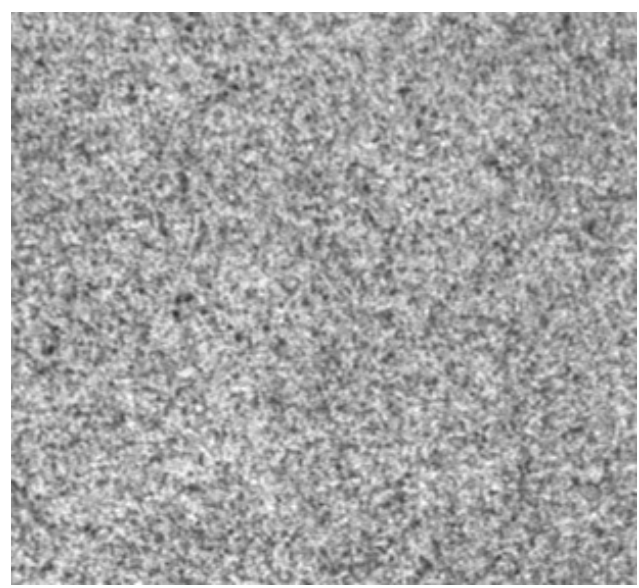

(b) after loading

Fig. 4 the images of crack-tip field

A part of the images before and after loading were elected as the measured field. The displacements of the measured field were obtained using white light digital image analysis in frequency domain and shown as follows.

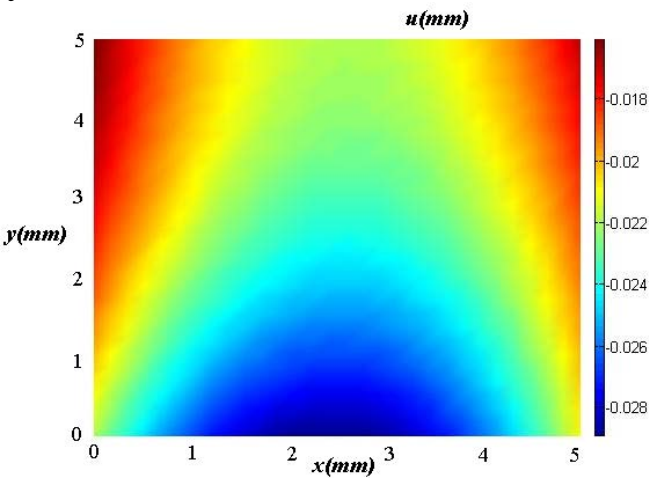

(a) $u$

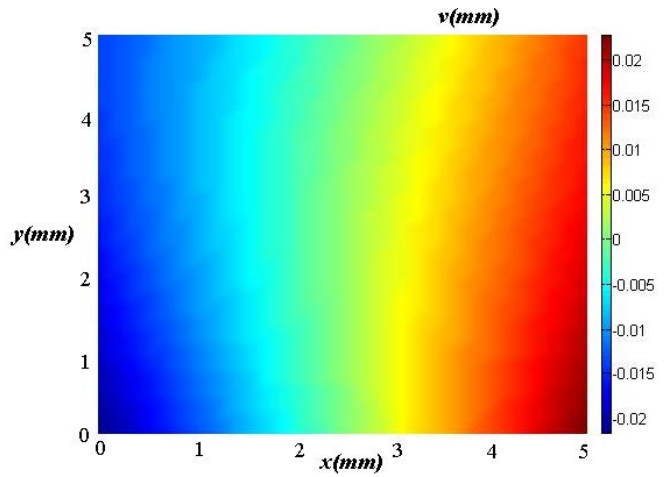

(b) $v$

Fig. 5 the displacements of measured field

The $\boldsymbol{u}$ displacements were chosen to calculate I type crack tip stress intensity factor $\boldsymbol{K}_{\mathrm{I}}$ according to the formula

$$
u=\frac{1+\mu}{2 E} K_{I} \sqrt{\frac{r}{2 \pi}}\left[(2 k-1) \cos \frac{\theta}{2}-\cos \frac{3 \theta}{2}\right],
$$

and we obtained the average value $K_{\mathrm{I}}=0.0546 \mathrm{MPa} \sqrt{m}$. Compared with the theoretical value $0.0517 \mathrm{MPa} \sqrt{\mathrm{m}}$, the error is enough little and the usability of white light digital image analysis in frequency domain is proved. 


\section{Conclusions}

In this paper, white light digital image analysis in frequency domain was introduced to study the fracture characteristics of machine-made sand concrete. The images of crack-tip field of the specimen before and after loading were recorded using CCD camera and input into computer. The displacements of measured field were obtained. and then I type crack tip stress intensity factor $K_{\mathrm{I}}$ was calculated. Compared the experimental results with the theoretical ones, the usability of white light digital image analysis in frequency domain for studying the fracture characteristics of machine-made sand concrete is certified.

\section{Acknowledgements}

The authors acknowledge the financial support of the Scientific Research Fund of Hebei Provincial Education Department, China(No. Z2014160)

\section{References}

[1]Tan Likun, Song Yupu and Chen Haoran, "Experimental study of strength and deformation of concrete under biaxial tension-compression stress after high temperature”, Engineering Mechanics, 2006, 23(2)112-116

[2] Gao Shuling, Xu Shilang, “Critical concrete crack length determination using strain gauges”, Journal of Tsinghua University (Science and Technology), 2007, 47(3):1432-1434

[3] Xinwei Yang, Xiumei Jin, Ruilan Tian, Study on Fracture Characteristics of Concrete by White Light DigitalImage Analysis in Frequency Domain, Applied Mechanics and Materials 2010, 29-32: 490-495

[4] Xinwei Yang, Honggao Man, Ruilan Tian, Deformation Measurement of Concrete by White Light Digital Image Analysis in Frequency Domain, Applied Mechanics and Materials 2010, 29-32: 496-501 